JORNADAS

\title{
HIPOGLUCEMIAS EN PACIENTES CON ENFERMEDAD RENAL CRÓNICA
}

\author{
HYPOGLYCEMIA IN PATIENT WITH CHRONIC KIDNEY DISEASE
}

\author{
Gabriela Medek
}

\section{RESUMEN}

El control glucémico estricto demostró disminuir la incidencia y progresión de las complicaciones crónicas, con el consecuente incremento del riesgo de hipoglucemias. Actualmente la Asociación Americana de Diabetes clasifica: Nivel 1: alarma de hipoglucemia $<70 \mathrm{mg} / \mathrm{dl}$; Nivel 2: hipoglucemia clínicamente significativa $<54$ mg/dl; Nivel 3: hipoglucemia severa en la cual, sin especificar el valor de glucemia, se requiere la ayuda de terceros o se presentan alteraciones cognitivas. La hipoglucemia suele ser menos frecuente en pacientes con diabetes tipo 2 que en diabetes tipo 1 ; se relaciona con alta morbilidad y mortalidad, y representa frecuentemente una de las principales barreras para lograr un óptimo control glucémico. Los pacientes con filtrado glomerular (FG) disminuido $\left(<60 \mathrm{ml} / \mathrm{min} / 1,73 \mathrm{~m}^{2}\right)$ frecuentemente presentan menores requerimientos de insulina y disminución de la degradación de la insulina en los tejidos periféricos, los cuales son factores de riesgo para el desarrollo de hipoglucemia severa.

Palabras clave: hipoglucemia, enfermedad renal crónica, morbilidad, mortalidad.

Revista de la Sociedad Argentina de Diabetes 2017; Vol. 51 (100-102)

\section{ABSTRACT}

The strict blood glucose monitoring has proved to reduce the incidence and progression of chronic complications, with the resulting increase of hypoglycemia risk. At present, the American Association of Diabetes classifies: Level 1: warning of hypoglycemia $<70 \mathrm{mg} / \mathrm{dl}$; Level 2: clinically significant hypoglycemia <54 mg/ dl; Level 3: severe hypoglycemia, in which without specifying the value of glycemia, it is necessary the help of third parties or cognitive alterations appear. Hypoglycemia is usually less frequent in patients with type 2 diabetes rather than type 1 diabetes; it is associated with the high morbidity and mortality, representing frequently one of the principal barriers to attain an optimal glycemia control. Patients with reduced glomerular filtration (GF) $(<60$ $\mathrm{ml} / \mathrm{min} / 1.73 \mathrm{~m}^{2}$ ) frequently present less insulin requirements and decrease of insulin degradation in peripheral tissue, which are risk factors for the development of severe hypoglycemia.

Key words: hypoglycemia, chronic kidney disease, morbidity, mortality.

Revista de la Sociedad Argentina de Diabetes 2017; Vol. 51 (100-102)
Especialista en Medicina Interna, especializada en Diabetes; Médica del Servicio de Diabetes y Nutrición en la Policlínica Bancaria; Consultorio de Diabetes, La Plata, Prov. de Bs. As.; miembro del Comité de Nefropatía de la Sociedad Argentina de Diabetes, CABA, Argentina

Contacto de la autora: Gabriela Medek

E-mail: gmedek@hotmail.com
Correspondencia: Acevedo $840 \quad 5^{\circ}$ piso, Depto. 16 (C1414DJR), CABA, Argentina

Tel: (011) 0221-155401061

Fecha de trabajo recibido: 18/08/17

Fecha de trabajo aceptado: 30/08/17

Conflictos de interés: la autora declara que no existe

conflicto de interés

\section{Hipoglucemias}

La diabetes mellitus (DM) es la causa más frecuente de enfermedad renal (ER) en el mundo. El control glucémico estricto es de gran importancia en estos pacientes ya que se ha demostrado en numerosos ensayos clínicos que disminuye la incidencia y progresión de las complicaciones micro y macrovasculares, así como también la evolución a la enfermedad renal crónica terminal1,2,3.

La American Diabetes Association (ADA) clasifica a la hipoglucemia:
- Nivel 1: alarma de hipoglucemia $<70$ mg/dl.

- Nivel 2: hipoglucemia clínicamente significativa $<54 \mathrm{mg} / \mathrm{dl}$.

- Nivel 3: hipoglucemia severa en la cual, sin especificar el valor de glucemia, se requiere la ayuda de terceros o se presentan alteraciones cognitivas ${ }^{4}$.

La hipoglucemia se relaciona con alta morbilidad y mortalidad, y representa frecuentemente una de las principales barreras para lograr un óptimo control glucémico.

Los eventos de hipoglucemia en pacientes 
con DM2 suelen ser menos frecuentes que en los pacientes con DM1, posiblemente debido a la preservación relativa de las células beta con respuestas hormonales contrarregulatorias en los pacientes con DM2 ${ }^{5}$. Los eventos agudos de hipoglucemia pueden resultar en un aumento de la actividad adrenérgica, isquemia miocárdica, arritmias cardíacas graves y muerte súbita. Esto explicaría, al menos en forma parcial, el mayor riesgo de enfermedad cardiovascular que estos pacientes presentan ${ }^{6,7}$.

Independientemente del tipo de diabetes y del tratamiento recibido, la presencia de enfermedad renal crónica (ERC) es uno de los principales factores de riesgo para el desarrollo de hipoglucemia severa. El riñón cumple un papel importante en la depuración de la insulina, razón por la cual los pacientes con DM y ERC con filtrado glomerular (FG) disminuido $\left(<60 \mathrm{ml} / \mathrm{min} / 1,73 \mathrm{~m}^{2}\right)$ frecuentemente presentan menores requerimientos de insulina; además, la degradación de la insulina en los tejidos periféricos disminuye en esta población de pacientes ${ }^{8}$. En situación de ERC y uremia coexiste frecuentemente una disminución de la ingesta, con una nutrición subóptima que conduce a un descenso en las reservas de glucógeno ${ }^{9}$. En estos pacientes la gluconeogénesis renal se encuentra disminuida ${ }^{8}$. Finalmente, los fármacos antidiabéticos más frecuentemente utilizados son excretados por vía renal y tienen una vida media prolongada en pacientes con ERC lo cual los predispone a episodios de hipoglucemia. Otros factores predisponentes son: la polifarmacia, edad avanzada, duración de la diabetes, historia de hipoglucemia previa, duración del tratamiento con insulina o neuropatía periféri$\mathrm{ca}^{10,11}$. La confluencia de estos factores puede contribuir a un mayor riesgo de hipoglucemias.

En Estados Unidos el National Health and Nutrition Examination Survey (NHANES) de 2011-2012 señaló que aproximadamente el 19\% de los participantes con DM tenía una tasa FG $<60 \mathrm{ml} / \mathrm{min} / 1,73 \mathrm{~m}^{2}$. La prevalencia de ERC, caracterizada por disminución del FG o presencia de albuminuria, resultó de aproximadamente el $50 \%{ }^{12}$. Un estudio realizado en Reino Unido de- mostró que los pacientes con diabetes tuvieron cuatro veces más probabilidades de tener ERC que aquellos sin diabetes $(31 \% \text { vs } 6,9 \%)^{13}$.

La incidencia y prevalencia exacta de hipoglucemia en pacientes con DM y/o ERC es difícil de definir ya que puede pasar desapercibida o no ser reportada; en general no se detecta en un $25 \%$ de los pacientes ${ }^{14}$.

La hipoglucemia renal (hipoglucemia asociada en ERC sin otra causa aparente) puede ocurrir espontáneamente en individuos con DM con una incidencia del 1-3\%. Moen et al. encontraron que la incidencia de hipoglucemia se incrementa en presencia de diabetes o ERC, siendo mayor el riesgo cuando se encuentran ambas. Entre los pacientes con diabetes, la tasa fue de 10,7 vs 5,3/100 pacientes/mes, mientras que en los pacientes sin DM fue de 3,46 vs 2,23/100 pacientes/mes, para la ERC vs no ERC respectivamente ${ }^{10}$. Mühlhauser et al. demostraron que los pacientes con DM1 y ERC tenían una incidencia cinco veces mayor de hipoglucemia severa que aquellos con DM1 y creatinina sérica normal' ${ }^{15}$.

En las personas con DM2 la frecuencia de hipoglucemias varía según la modalidad de tratamiento y es mayor en los pacientes que reciben insulina o secretagogos de insulina excretados principalmente por el riñón o con metabolitos activos que pueden acumularse en los pacientes con insuficiencia renal ${ }^{16}$. La insulina prandial de acción rápida se asocia con mayor frecuencia de hipoglucemia que la insulina basal de acción prolongada. La metformina, las tiazolidinedionas, los inhibidores de la dipeptidil peptidasas-4, los miméticos del péptido 1 similar al glucagón (GLP-1) y los inhibidores del cotransportador sodio glucosa tipo 2 (SGLT-2) no aumentan el riesgo de hipoglucemia cuando se usan sin insulina o sulfolinureas $17,18,19$.

Durante un período de seguimiento de 10 años, Yun et al. demostraron que la presencia de excreción urinaria de albumina $>300$ mg/día fue un factor de riesgo independiente para el desarrollo futuro de hipoglucemia severa en pacientes con DM2 con función renal normal o mínimamente disminuida. El mecanismo por el 
cual se produce este fenómeno se desconoce ${ }^{11}$. En los últimos años se jerarquizó la presencia de hipoglucemia como un factor limitante en el tratamiento de la DM debido a su asociación con aumento del riesgo de morbimortalidad cardíaca.

Si se considera que los pacientes con ERC presentan elevado riesgo cardiovascular, un objetivo primario de tratamiento sería evitar los episodios de hipoglucemias. Por otro lado, la ER es un factor de riesgo de las mismas debido a varios mecanismos (disminución de la depuración de insulina, la gluconeogénesis, el metabolismo y depuración de drogas, y produce disminución de la ingesta); esta situación se incrementa a medida que progresan los estadios de la ER, más aún en presencia de insuficiencia y/o tratamiento sustitutivo. Por lo tanto debe considerarse lo dicho anteriormente para establecer los objetivos glucémicos y el tratamiento en los pacientes con DM y ERC.

\section{BIBLIOGRAFÍA}

1. Luboswsky ND, Siegel R, Pittas AG, et al. Manegment of glycemia in patients with diabetes mellitus and CDK. Am J Kidney Dis 2007; 50(5):865-879.

2. UK Prospective Diabetes Study (UKPDS) Group. Intensive blood-glucose control with sulphonylureas or insulin compared with conventional treatment and risk of complications in patients with type 2 diabetes (UKPDS 33). Lancet 1998; 352(9131): 837-53.

3. Khaw KT, Wareham N, Luben R, et al. Glycated haemoglobin, diabetes, and mortality in men in Norfolk cohort of european prospective investigation of cancer and nutrition (EPIC-Norfolk). BMJ 2001; 322(7277):15-18.

4. Standars of Medical Care. American Diabetes Association. Glycemic targets. Diabetes Care 2017; 40(Suppl. 1): S48-S56.

5. Akram K, Pedersen-Bjergaard U, Borch-Johnsen K. Frecuency and risk factors of severe hypoglycemia in insulin treated type 2 diabetes: a literatura survey. J Diabetes Complications 2006; 20(6): 402-408.
6. The ADVANCE Collaborative Group. Intensive blood glucose control and vascular outcomes in patients with type 2 diabetes. N Engl J Med 2008; 358(24): 2560-72.

7. Robinson RT, Harris ND, Ireland RH, et al. Mechanisms of abnormal cardiac repolarization during insulin-induced hypoglycemia. Diabetes 2003; 52(6): 1469-74.

8. Biesenbach G, Raml A, Schemeckal B, et al. Decreased insulin requirement in relation to GRF in nephopatic type 1 and insulin- treated type 2 diabetic patients. Diabet Med 2003; 20(8): 642-5.

9. Snyder RW, Bernds JS. Use of insulin and oral hypoglycemic medications in patients with diabetes mellitus and advanced kidney desease. Semin Dial 2004; 17(5): 665-70.

10. Moen MF, Zhan M, Hsu VD, et al. Frequency of hypoglycemia and its significance in chronic kidney disease. Clin J Am Soc Nephrol 2009; 4(6):1121-7.

11. Jae-Seung Y, Sun-Hye K, Sun-Hee K, et al. Presence of macroalbuminuria predicts severe hypoglycemia in patients with type 2 diabetes. Diabetes Care 2013; 36(5): 1283-89.

12. Collins AJ, Foley RN, Herzog C, et al. US Renal Data System 2012 Annual Data Report. Am J Kidney Dis 2013; 61 (1 Suppl 1): e1-e476.

13. New JP, Middleton RJ, Klebe B, et al. Assessing the prevalence, monitoring and management of chronic kidney disease in patients with diabetes compared with those without diabetes in general practice. Diabet Med 2007; 24(4): 364-9.

14. Gerich JE, Mokan M, Veneman T, et al. Hypoglycemia unawareness. Endocr Rev 1991; 12(4):356-71.

15. Mühlhauser I, Toth G, Sawicki PT, et al. Severe hypoglycemia in type I diabetic patients with impaired kidney function. Diabetes Care 1991; 14(4): 344-346.

16. Zammitt NN, Frier BM. Hypoglycemia in type 2 diabetes: pathophysiology, frequency, and effects of different treatment modalities. Diabetes Care 2005; 28(5): 2948-61.

17. Amiel SA, DixonT, Mann R. Hypoglucemia in type 2 diabetes. Diabet Med 2008; 25(3):245-54.

18. Taylor JR, Dietrich E, Powell JG. New and emerging pharmacologic therapies for type 2 diabetes, dyslipidemia, and obesity. Clin Ther 2013; 35: A3-A17.

19. Holman RR, Farmer AJ, Davies MJ, et al.Three-year efficacy of complex insulin regimens in type 2 diabetes. $\mathrm{N}$ Engl J Med 2009; 361(18):1736-47. 